\title{
Culturally adapted depression education and engagement in treatment among Hispanics in primary care: outcomes from a pilot feasibility study
}

Katherine Sanchez ${ }^{1,2^{*}}$ (D) Michael O. Killian ${ }^{3}$, Brittany H. Eghaneyan ${ }^{1}$, Leopoldo J. Cabassa ${ }^{4}$ and Madhukar H. Trivedi ${ }^{2}$

\begin{abstract}
Background: Low use of anti-depressant medication, poor doctor-patient communication, and persistent stigma are key barriers to the treatment of depression in Hispanics. Common concerns include fears about the addictive and harmful properties of antidepressants, worries about taking too many pills, and the stigma attached to taking medications and seeking mental health treatments. In 2014, the Center for Medicare and Medicaid Services (CMS) funded the Depression Screening and Education: Options to Reduce Barriers to Treatment (DESEO) project to implement an education intervention designed to increase disease literacy and dispel myths about depression and its treatment among Hispanic patients thus reducing stigma and increasing treatment engagement.
\end{abstract}

Methods: The DESEO study utilized a one-group pretest-posttest design to assess the effects a culturally-adapted Depression Education Intervention's (DEI) on depression knowledge, stigma, and engagement in treatment in a sample of 350 Hispanic primary care patients with depression. The DEl utilized a fotonovela, a health education tool available in English and Spanish that uses posed photographs, captions, and soap opera narratives to raise awareness about depression and depression treatments.

Results: Participants reported significant decreases in depression symptoms and reported stigma about mental health care. Additionally, participants reported increased knowledge of depression yet greater negative perceptions about antidepressant medication. Finally, 89.5\% of participants reported entering some form of treatment at follow-up.

Conclusions: Culturally adapted depression education shows promise in increasing understanding of depression, decreasing stigma, and increasing treatment engagement among Hispanic patients in a community-based health center. Results have implications for practice in addressing common concerns about depression treatments which include fears about the addictive and harmful properties of antidepressants, worries about taking too many pills, and the stigma attached to taking psychotropic medications.

Trial registration: The study was retrospectively registered with www.clinicaltrials.gov: NCT02491034 July 2, 2015. Keywords: Depression, Education, Hispanics, Stigma, Primary care, Fotonovela

\footnotetext{
* Correspondence: KSanchez@uta.edu

${ }^{1}$ School of Social Work, University of Texas at Arlington, 211 South Cooper

Street, Arlington, TX 76019, USA

2Department of Psychiatry, UT Southwestern Medical Center, Dallas, TX, USA

Full list of author information is available at the end of the article
}

(c) The Author(s). 2019 Open Access This article is distributed under the terms of the Creative Commons Attribution 4.0 International License (http://creativecommons.org/licenses/by/4.0/), which permits unrestricted use, distribution, and reproduction in any medium, provided you give appropriate credit to the original author(s) and the source, provide a link to the Creative Commons license, and indicate if changes were made. The Creative Commons Public Domain Dedication waiver (http://creativecommons.org/publicdomain/zero/1.0/) applies to the data made available in this article, unless otherwise stated. 


\section{Background}

The World Health Organization estimates mental disorders collectively account for more than $13 \%$ of the global burden of disease from all causes, are the leading cause of disability and associated with the highest rates of unemployment of all disabilities [1]. One in six Americans will experience depression in their lifetime, and recurrence is common, costing an estimated $\$ 83-125$ billion in the U.S. each year, more than half of which is publicly funded [2,3]. Virtually all Medicare spending growth in recent years is associated with patients who were treated for five or more conditions, including depression [3].

Individuals with depression are at greater risk for both cardiovascular diseases and type II diabetes [4]. Adequately treating patients with depression and comorbid chronic medical illnesses, like diabetes, can improve morbidity and decrease costs. Safety-net public mental health resources are at capacity, and there remains a significant unmet need [5]. Primary care is well-positioned to deliver services for common mental disorders and, because of insufficient capacity of both primary and specialty care, Federally Qualified Health Centers (FQHCs) play an essential role in promoting access to preventive and primary care among medically underserved populations, including Medicaid, Medicare and the Children's Health Insurance Program (CHIP) enrollees [6].

Hispanics residing in the United States experience mental health disorders at a rate of $28.1 \%$ for men and $30.2 \%$ for women [7]. Factors associated with diagnosis of a psychiatric disorder among Hispanic populations include being born in the United States and English proficiency [8]. Hispanics have a higher prevalence of diabetes and comorbid depression which may take significantly longer to treat effectively $[9,10]$. Depression relapse rates are high and response to treatment is often slow among Hispanic populations, which often results in the discontinuation of medication [10]. Hispanics experience a considerable burden of disease compared to nonHispanic whites [10, 11].

Lack of uptake in anti-depressant medication use and stigma, in conjunction with poor communication, are key barriers to depression treatment in Hispanic populations [10, 12, 13]. Common concerns about treatment include fears about the addictive and harmful properties of antidepressants, worries about taking too many pills, and the stigma attached to taking medications [14-16]. Additional barriers include lack of insurance, costs of medications, absence of Spanish-speaking staff, and concerns about immigration status $[17,18]$.

The Center for Medicare and Medicaid Services (CMS) funded the Depression Screening and Education: Options to Reduce Barriers to Treatment (DESEO) project to implement a depression education intervention among Hispanic patients with the goal of reducing stigma and increasing treatment engagement [19]. In this analysis of the primary outcomes from DESEO we sought to examine, does the introduction of a culturally-adapted education intervention after screening positive for depression improve knowledge of the disorder and reduce stigma? Additionally, does the Depression Education Intervention (DEI) lead to subsequent engagement in treatment (any kind) for depression? Our hypothesis was that the provision of culturally-adapted depression education to Hispanic patients in a community-based health center would lead to an increase in engagement in treatment.

\section{Methods}

\section{Study design and setting}

The DESEO study utilized a one-group pretest-posttest design to assess a culturally-adapted DEI effects on depression knowledge, stigma, and engagement in treatment within a sample of Hispanic primary care patients diagnosed with depression [19]. The study took place in an FQHC in a large metropolitan area in Texas between February 2015 and October 2016. The community clinic provides comprehensive primary care to low-income, primarily Hispanic populations. Behavioral health services are provided by a Licensed Clinical Social Worker (LCSW) and include brief counseling using evidencebased interventions such cognitive behavioral therapy and behavioral activation. The study was reviewed and approved by the Institutional Review Board of the University of Texas at Arlington.

\section{Sample}

From February 2015 through October 2016, all adult primary care patients were universally screened for depression using the Patient Health Questionnaire-9 (PHQ-9) [20], during annual or new/non-acute visits. Patients who were 18 years or older, self-identified as Hispanic, met diagnostic criteria for depression, and were not already receiving treatment were invited to participate in the study [19]. The final study sample included 350 Hispanic participants. For further detail on sample acquisition see Lopez, et al. [21]

\section{Intervention}

The DEI offered to all participants utilized a comic-book style pamphlet titled "Secret Feelings" developed by Cabassa, Molina, and Baron [22]. The fotonovela was presented in both English and Spanish at a 4th grade reading level and uses an entertainment-education approach to portray the story of a Latina woman experiencing depression as she describes her symptoms, presents common fears and misconceptions (addiction, sexual dysfunction), and displays both informal and formal help-seeking behaviors (a friend and a trusted 
pharmacist) as she discusses her mental health concerns with her family and engages in formal depression treatment $[21,23]$. The fotonovela has been tested via two randomized trials in clinical and non-clinical settings demonstrating its effectiveness to decrease stigma toward mental health care and increase knowledge of depression [23-25].

The clinic's bilingual LCSW served as the Depression Educator (DE) and read the fotonovela with the participants, using it to promote discussion centered around the participants' own experiences with depression, treatment options, and stigmas and fears concerning potential treatments. The DEI was delivered in English or Spanish and in the presence of family members or loved ones, if desired. At the conclusion of the discussion, the DE invited participants to participate in the decisionmaking process for their own depression treatment and offered treatment options including behavioral activation strategies (walking, gardening, visiting with friends), counseling with the $\mathrm{DE}$, and/or antidepressant medication. The DE could facilitate a warm hand off back to the provider if the patient expressed an interest in antidepressants and the primary care provider could prescribe the medication.

\section{Procedures}

Universal screening for depression was implemented for all adult patients during visits with their primary care providers using the 9-item Patient Health Questionnaire (PHQ-9) [20]. The DE administered four additional baseline measures including the Depression Knowledge Measure (DKM) [25] and three stigma measures (see below). All informed consent documents and measures were offered in English or Spanish based on patient preference. Detailed procedures for the study can be found in Sanchez, et al. [19]

When the participants returned for their DEI session, the DE administered the PHQ-9 prior to the education intervention, and the DKM and three stigma measures afterwards. One month after the DEI session, participants were contacted by the $\mathrm{DE}$ or a student research assistant to complete the final measures by phone including the PHQ-9, DKM, three stigma measures, and to assess whether they had engaged in treatment. See Fig. 1 for study flow diagram.

\section{Measures}

\section{Depression}

Depression severity was measured using the PHQ-9, a self-report measure that assesses the frequency of depression symptoms within the last two weeks using each of the of the nine Diagnostic and Statistical Manual of Mental Disorders (DSM-IV) [26] criteria for depression. Total scores range from 0 to 27 with a PHQ-9 score $\geq 10$ are considered clinically significant depressive symptoms. The PHQ-9 has demonstrated to be a reliable and valid measure of depression severity in racially and ethnically diverse primary care samples [27].

\section{Depression knowledge}

The DKM was developed by Unger et al. [25] to evaluate the effect of the fotonovela, Secret Feelings, on depression knowledge. The measure has a total of 17 items including symptom recognition of five DSM depression symptoms (sleeping too little, eating too much, feeling agitated, feeling guilty, and loss of interest) and five nondepressive symptoms (hearing voices, being full of energy, being violent, having hallucinations, and feeling confident). One point was allocated for each correct response, with total scores ranging from 0 (all incorrect) to 17 (all correct).

\section{Stigma}

Three measures developed by Interian et al. [28] were used to assess depression stigma. The Stigma Concerns about Mental Health Care (SCMHC) scale is a 3-item scale (possible scores ranging from 0 to 3 ) used to measure stigma towards depression treatment. The SCMHC has demonstrated internal consistency with a Cronbach's alpha of 84 .

The Latino Scale for Antidepressant Stigma (LSAS) includes seven items measuring stigma towards the use of antidepressant medications. Possible scores for the scale range from 0 to 14, with higher scores indicating greater stigma. The LSAS has demonstrated internal consistency with a Cronbach's alpha of .80 .

Finally, the Social Distance (SD) scale was used to measure social distance desirability from someone with depression or history of depression treatment $[29,30]$. The measure has six items with lower scores indicating greater desired social distance (i.e., greater stigma). The SD has demonstrated internal consistency with a Cronbach's alpha of .75 .

\section{Treatment engagement}

Participants self-reported whether they had engaged in treatment for their depression at the one-month followup phone call by responding yes/no to the question "Since meeting with the DE and discussing the fotonovela, have you participated in some type of treatment for your depression?" If the patient endorsed "yes" they were engaged in treatment, additional response choices were collected and included 1) taking medication only, 2) receiving counseling only, 3) taking medication and receiving counseling, and 4) other behavioral interventions. "Taking medication" was defined as currently taking medication prescribed to treat depression. "Receiving counseling" was defined as attending at least one 


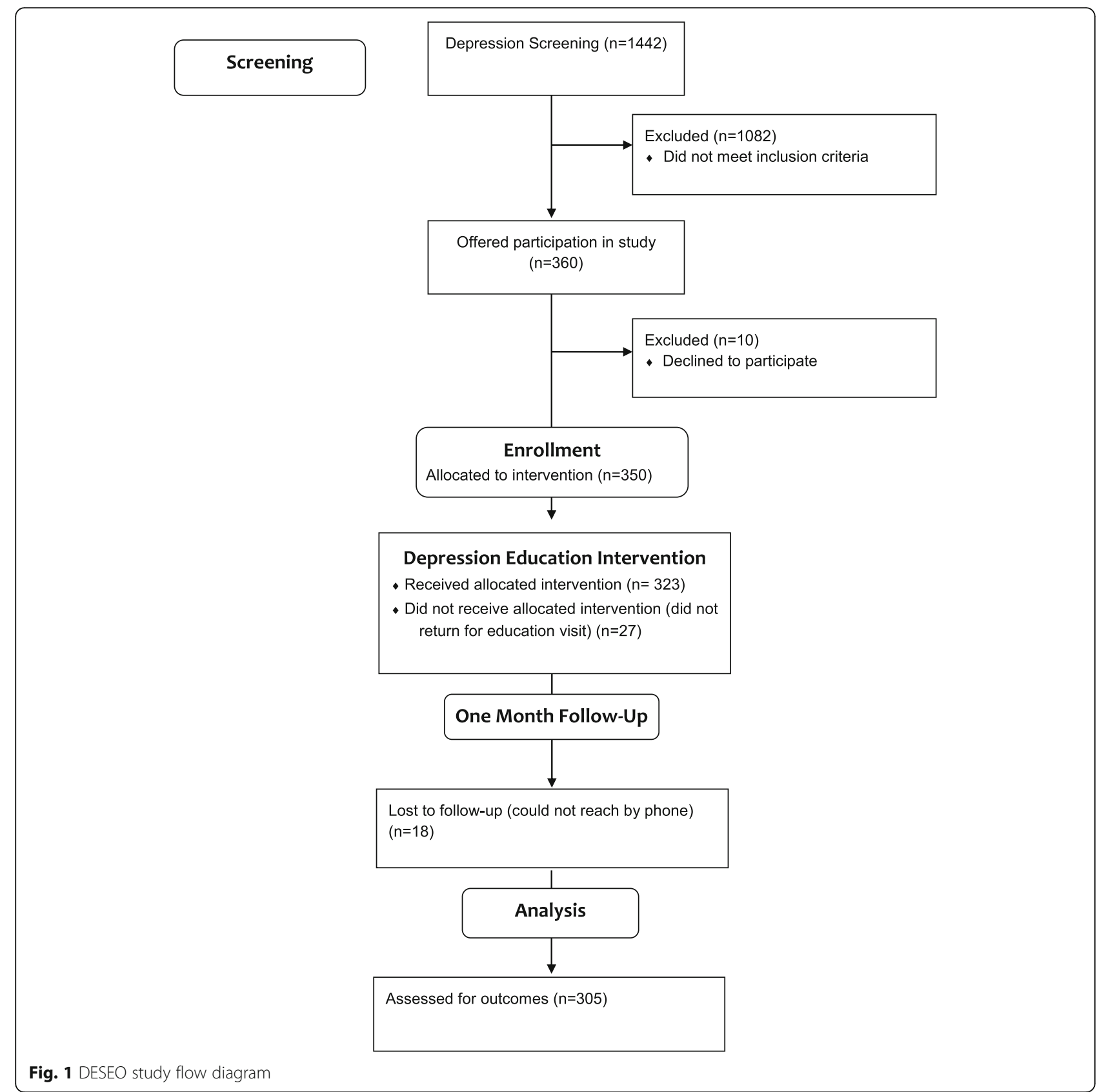

counseling session with the DE or other mental health professional after the DEI session. "Other behavioral interventions" included behavioral activation (BA) activities that the DE and the participant set as goals for the treatment of their depression and responses were collected qualitatively.

\section{Statistical analyses}

Testing for differences between the group completing the protocol and those participants dropping out of the study (attritioners) used various bivariate statistical tests (Table 1). Testing for changes in participant reported scores over time used repeated-measures analysis of variance (RMANOVA). Multinomial logistic regression was used to test the association between the self-report measures from follow-up and the likelihood of the treatment engagement categories (i.e., no treatment as the reference category, counseling only or other behavioral interventions, and medication or medication with counseling). Effect sizes were calculated and included partial $\eta^{2}$ and Nagelkerke $r^{2}$ All tests assumed a significance value of .05 and were completed using SPSS 25.0 software (IBM, 2017). 
Table 1 Descriptive statistics of sample

\begin{tabular}{|c|c|c|c|c|c|}
\hline & Total Sample $(n=350)$ & Completers $(n=305)$ & Attritioners $(n=45)$ & Test & $p$-value \\
\hline Age, $M(S D)$ & $38.81(10.60)$ & $39.00(10.00)$ & $37.53(14.08)$ & $t=0.676$ & .502 \\
\hline Gender, female, $n(\%)$ & $327(93.4 \%)$ & $285(93.4 \%)$ & $42(93.3 \%)$ & Fisher Exact & .999 \\
\hline Spanish Speaking, yes, n (\%) & $332(95.1 \%)$ & $288(94.7 \%)$ & $44(97.8 \%)$ & Fisher Exact & .708 \\
\hline Marital Status, $n(\%)$ & & & & $x^{2}=4.289$ & .368 \\
\hline Married/cohabitating & $247(70.6 \%)$ & $217(71.1 \%)$ & $30(66.7 \%)$ & & \\
\hline Never married & $39(11.1 \%)$ & $36(11.8 \%)$ & $3(6.7 \%)$ & & \\
\hline Widowed & $10(2.9 \%)$ & $8(2.6 \%)$ & $2(4.4 \%)$ & & \\
\hline Divorced & $29(8.3 \%)$ & $25(8.2 \%)$ & $4(8.9 \%)$ & & \\
\hline Other & $25(7.1 \%)$ & $19(6.2 \%)$ & $6(13.3 \%)$ & & \\
\hline Education Level, $n$ (\%) & & & & $X^{2}=2.014$ & .847 \\
\hline 8 th grade or less & $126(36.8 \%)$ & $111(36.4 \%)$ & $15(40.5 \%)$ & & \\
\hline Some high school & $92(26.9 \%)$ & $83(27.2 \%)$ & $9(24.3 \%)$ & & \\
\hline High school or GED & 75 (21.9\%) & $67(22.0 \%)$ & $8(21.6 \%)$ & & \\
\hline Vocational or trade school & $9(2.6 \%)$ & $7(2.3 \%)$ & $2(5.4 \%)$ & & \\
\hline Some college & $30(8.8 \%)$ & $28(9.2 \%)$ & $2(5.4 \%)$ & & \\
\hline College degree & $10(2.9 \%)$ & $9(3.0 \%)$ & $1(2.7 \%)$ & & \\
\hline Patient Health Questionnaire, M (SD) & $17.55(3.89)$ & $17.53(3.70)$ & 17.69 (4.99) & $t=0.206$ & .838 \\
\hline No depression, $n$ (\%) & $0(0 \%)$ & $0(0 \%)$ & $0(0 \%)$ & $X^{2}=2.502$ & .475 \\
\hline Mild depression, $n$ (\%) & $7(2.0 \%)$ & $5(1.6 \%)$ & $2(4.4 \%)$ & & \\
\hline Moderate depression, $n(\%)$ & $66(18.9 \%)$ & $57(18.7 \%)$ & $9(20.0 \%)$ & & \\
\hline Moderately severe depression, $n$ (\%) & $167(47.7 \%)$ & $149(48.9 \%)$ & $18(40.0 \%)$ & & \\
\hline Severe depression, $n$ (\%) & $110(31.4 \%)$ & $94(30.8 \%)$ & $16(35.6 \%)$ & & \\
\hline Depression Knowledge Measure, M (SD) & $10.76(2.15)$ & $10.81(2.15)$ & $10.44(2.15)$ & $t=1.053$ & .293 \\
\hline Stigma Concerns About Mental Health Care, M (SD) & $.44(.85)$ & $.43(.84)$ & $.47(.89)$ & $t=0.239$ & .811 \\
\hline Latino Scale for Antidepressant Stigma, M (SD) & $6.13(3.45)$ & $6.12(3.42)$ & $6.18(3.72)$ & $t=0.107$ & .915 \\
\hline Social Distance, $M(S D)$ & $9.00(3.07)$ & $9.05(2.99)$ & $8.64(3.61$ & $t=0.831$ & .407 \\
\hline \multicolumn{6}{|l|}{ Treatment Outcome and Engagement, $n$ (\%) } \\
\hline Medication or counselling with medication & $106(34.8 \%)$ & - & - & & \\
\hline Counseling or other behavioral intervention & $167(54.8 \%)$ & - & - & & \\
\hline No treatment at follow-up & $32(34.8 \%)$ & - & - & & \\
\hline
\end{tabular}

\section{Results}

\section{Sample characteristics}

At baseline, nearly half of the sample participants $(N=$ 350) reported moderately severe depression scores $(n=$ $167,47.7 \%$ ) with only $2 \%$ of the sample reporting less than moderate depression. Severe depression scores were reported by $31.4 \%$ of the sample $(n=110)$, but there were no reported adverse events or psychiatric emergencies related to suicidal ideation or self-harming. The sample was nearly all women $(n=327,93.4 \%)$ with a mean age of 38.81 years $(\mathrm{SD}=10.60$, range 18 to 82 years). The sample was entirely Hispanic and their country of origin unknown, though $88 \%$ of Hispanics in Texas are of Mexican descent [31]. See Table 1. No significant differences were found between those completing the protocol $(n=305,87.1 \%)$ and those who dropped out $(n=45,12.9 \%)$ based on their demographic characteristics or baseline measurement in the study (Table 1).

\section{Self-report measures: changes over time}

The descriptive statistics for the primary outcomes related to changes in stigma and depression knowledge are provided in Table 2 for those participants who completed the protocol $(n=305)$. Stigma Concerns About Mental Health Care (F $[1.907$, 574.076] $=21.914$, $p<.001$, partial $\left.\eta^{2}=.068\right)$ significantly decreased over time. Similarly, social distance scores significantly increased over time $(\mathrm{F}[1.823,548.637]=50.288, p<.001$, partial $\eta 2=.143$ ) indicating less desire for social distance 
Table 2 Changes in self-reported measures over time $(n=305)$

\begin{tabular}{|c|c|c|c|c|c|}
\hline & Baseline & 2nd Session & One month follow-up & $\mathrm{F}$ & Partial $\eta^{2}$ \\
\hline Depression Knowledge Measure, M (SD) & $10.81(2.15)$ & $15.71(1.70)$ & $14.99(1.82)$ & $807.305^{* * *}$ & .730 \\
\hline Stigma Concerns About Mental Health Care, M (SD) & $.43(.84)$ & $.27(.67)$ & $.10(.43)$ & $21.914^{* * *}$ & .068 \\
\hline Latino Scale for Antidepressant Stigma, M (SD) & $6.12(3.42)$ & $6.56(3.46)$ & $7.19(2.62)$ & $14.633^{* * *}$ & .047 \\
\hline Social Distance, $M(S D)$ & $9.05(2.99)$ & $9.92(2.61)$ & $10.52(2.24)$ & $50.288^{* * *}$ & .143 \\
\hline Patient Health Questionnaire, M (SD) & $17.53(3.70)$ & $14.77(5.11)$ & $9.47(6.04)$ & $319.328^{* * *}$ & .516 \\
\hline No depression, $n$ (\%) & $0(0 \%)$ & $10(3.3 \%)$ & $77(25.2 \%)$ & - & \\
\hline Mild depression, $n$ (\%) & $5(1.6 \%)$ & $44(14.4 \%)$ & $89(29.2 \%)$ & - & \\
\hline Moderate depression, $n$ (\%) & $57(18.7 \%)$ & $82(26.9 \%)$ & $69(22.6 \%)$ & - & \\
\hline Moderately severe depression, $n$ (\%) & $149(48.9 \%)$ & $116(38.0 \%)$ & $54(17.7 \%)$ & - & \\
\hline Severe depression, $n$ (\%) & $94(30.8 \%)$ & $50(16.4 \%)$ & $16(5.2 \%)$ & - & \\
\hline
\end{tabular}

*** $p<.001$

from those with mental health disorders and less stigma, as did depression knowledge (F $[1.886,563.768]=$ 807.305, $p<.001$, partial $\eta 2=.730$ ). Latino Scale for Antidepressant Stigma scores significantly increased over time $\left(\mathrm{F}[2,588]=14.633, p<.001\right.$, partial $\left.\eta^{2}=.047\right)$ indicating greater concerns about others' negative perception of the use of psychiatric medication (Table 2).

Analysis of depression scores indicated participants reported significantly lower scores on the PHQ-9 (F $[1.876,562.718]=319.328, p<.001$, partial $\left.\eta^{2}=.516\right)$ at each time point. Effect sizes (partial $\eta^{2}$ ) indicated moderate changes in scores. At baseline, $79.7 \%(n=243)$ of the sample reported moderately severe or severe depression, and less than a quarter of the sample reported similar depression scores at follow-up $(n=70,22.9 \%)$. A majority of the sample reported no or mild depression at follow-up ( $n=166,54.4 \%)$ when only five participants reported these levels at baseline (1.6\%) (Table 2).

\section{Treatment engagement}

A majority of participants reported entering some form of treatment at follow-up ( $n=273$ of 305, 89.5\%). Almost $20 \%$ of participants $(n=58)$ reported attending some form of counseling without medication while a third reported either being prescribed medication or a combination of medication and counseling $(n=106$, $34.8 \%)$. Another third of the sample $(n=109,35.7 \%)$ reported engaging in BA activities which included dietary changes, exercise, meditation, yoga, and spending more time socializing with loved ones. Only about $10 \%$ of the sample reported not entering treatment following the intervention $(n=32,10.5 \%)$.

Self-reported treatment engagement at 30-day follow-up was coded as taking medication or counseling with medication, counseling only or other behavioral interventions, or reporting not engaged in any treatment (Table 3). At 30-day follow-up after the education intervention, PHQ-9
$\left(X^{2}=34.98, \mathrm{df}=2, p<.001\right.$, Nagelkerke $\left.\mathrm{r}^{2}=.128\right)$ and DKM scores $\left(X^{2}=11.23, \mathrm{df}=2, p=.004\right.$, Nagelkerke $\left.\mathrm{r}^{2}=.043\right)$ significantly predicted likelihood of treatment engagement. Specifically, for every one-point increase in depression knowledge scores, participants were $28.8 \%$ more likely to engage in counseling $(\beta=.253$, Wald $t=7.589, \mathrm{df}=$ $1, p=.006, \mathrm{OR}=1.288)$ and $40.9 \%$ more likely to report taking medication $(\beta=.343$, Wald $\mathrm{t}=11.235, \mathrm{df}=1$, $p=.001, \mathrm{OR}=1.409)$. A one-point increase in DKM score represents correct identification of a depression symptom or knowledge of the disorder, and greater overall knowledge. Participants with higher depression scores at 30-day follow-up were more likely to report engaging in medication treatment than those participants reporting no treatment engagement at follow-up $(\beta=.102$, Wald $t=8.122$, $\mathrm{df}=1, p=.004, \mathrm{OR}=1.108$ ).

After controlling for depressions scores at the 30-day follow-up, depression knowledge still predicted treatment engagement in a multivariate model. Participants were $28.6 \%$ more likely to engage in counseling $(\beta=.252$, Wald $\mathrm{t}=7.210, \mathrm{df}=1, p=.007, \mathrm{OR}=1.286)$ and $49.7 \%$ more likely to report taking medication $(\beta=.403$, Wald $\mathrm{t}=14.066, \quad \mathrm{df}=1, \quad p<.001, \quad \mathrm{OR}=1.497)$ compared to those who reported no treatment engagement (Table 3).

\section{Discussion}

Management of depression among Hispanics in community-based clinics is confounded by issues of disease literacy, cultural treatment preferences and financial barriers to care $[21,32,33]$. The culturally-adapted depression education fotonovela delivered by an LCSW in this primary care study appeared to increase knowledge of the disorder, reduced some stigma indicators, particularly related to mental health care use and desire for social distance from people with mental disorders, and lead to significant engagement in treatment for depression. The unexpected finding related to increased stigma 
Table 3 Bivariate and multivariate multinomial regression modeling of treatment engagement predicted by 30-day follow up scores $(n=305)$

\begin{tabular}{|c|c|c|c|c|c|c|c|c|c|c|c|c|}
\hline & \multirow[t]{2}{*}{ Model $X^{2}$} & \multirow[t]{2}{*}{ Nagelkerke $r^{2}$} & \multicolumn{5}{|c|}{ Counseling + Behavioral Intervention $^{a}$} & \multicolumn{5}{|c|}{ Medication or Meds+Counseling ${ }^{a}$} \\
\hline & & & $\bar{\beta}$ & Wald t & OR & $\begin{array}{l}95 \% \mathrm{Cl} \\
\text { lower }\end{array}$ & $\begin{array}{l}95 \% \mathrm{Cl} \\
\text { upper }\end{array}$ & $\bar{\beta}$ & Wald $t$ & OR & $\begin{array}{l}95 \% \mathrm{Cl} \\
\text { lower }\end{array}$ & $\begin{array}{l}95 \% \mathrm{Cl} \\
\text { upper }\end{array}$ \\
\hline \multicolumn{13}{|l|}{ Bivariate $^{a}$} \\
\hline PHQ-9 & $34.98^{* * *}$ & .128 & -.025 & .525 & .975 & .912 & 1.043 & .102 & $8.122^{* *}$ & 1.108 & 1.032 & 1.188 \\
\hline DKM & $11.23^{* *}$ & .043 & .253 & $7.589^{* *}$ & 1.288 & 1.076 & 1.542 & .343 & $11.235^{* *}$ & 1.409 & 1.153 & 1.723 \\
\hline SCMHC & 2.684 & .010 & -.386 & 1.375 & .680 & .356 & 1.296 & -.678 & $2.717^{+}$ & .508 & .227 & 1.137 \\
\hline LSAS & 677 & .003 & .015 & .037 & 1.015 & .874 & 1.178 & -.025 & .098 & .975 & .835 & 1.139 \\
\hline SD & .367 & .001 & .020 & .058 & 1.020 & .869 & 1.197 & .047 & .290 & 1.048 & .883 & 1.243 \\
\hline Multivariate $^{a b}$ & $49.305^{* * *}$ & .177 & & & & & & & & & & \\
\hline PHQ-9 & & & .252 & $7.210^{* *}$ & 1.286 & 1.070 & 1.546 & .403 & $14.066^{* * *}$ & 1.497 & 1.212 & 1.848 \\
\hline DKM & & & -.011 & .093 & .989 & .924 & 1.059 & .123 & $10.964^{* *}$ & 1.131 & 1.051 & 1.216 \\
\hline
\end{tabular}

toward antidepressant use may reflect the knowledge gained about sexual dysfunction and other side effects, and awareness of stigmatizing attitudes toward medication in general. These findings warrant further exploration, perhaps qualitatively, as side effects pose considerable challenges to treatment, often lead to the use of subtherapeutic doses, poor treatment adherence and quality of life [34].

While two previous randomized trials of "Secret Feelings" have produced improvements in depression knowledge and reductions in stigma indicators particularly stigma toward mental health care in nonclinical, adult education classes and in a community center among Hispanic adult women at risk for depression but not receiving mental health care [23-25], this is the first application of the depression fotonovela in primary care with patients diagnosed with clinical depression. The fotonovela concept has been widely recognized as an effective public health tool among Hispanics in the US and Latin America $[35,36]$ and recommended as a strategy for encouraging participation of Hispanics in research [37] however, its efficacy as a patient education intervention to improve depression literacy and treatment engagement in clinical settings has not been tested.

Shared decision making requires providers assess the patient's interest in initiating treatment, provide information on the risks and benefits of specific treatments in an understandable format, and discuss with patients their choices [38]. For patients struggling with stigma and fears about psychotropic medications, communication can be improved by adopting patientcentered approaches, which are essential to improve the quality of treatment and may improve outcomes [37, 39, 40].

Patients play a critical role in self-management of their illnesses and educating them to understand the behavioral and psychosocial elements of managing their disease is the first step to help improve opportunities for optimal health [41]. Our findings indicated a significant relationship between knowledge gained and subsequent engagement in counseling, adding to the evidence which suggests that decision aids, such as culturally-appropriate patient education can improve people's knowledge regarding options, and reduce their decisional conflict related to feeling uninformed and unclear about their personal values [42].

This study has some limitations. The funding opportunity from the CMS Hispanic Health Services Research Grant Program specified that funded projects be for educational intervention studies, which would inform populations-at-risk and that the intervention reach a minimum of 350 participants. This requirement made a comparison group from the same site impossible and introduced the limitations of a one-group, pretest-posttest design [19] There was also only one LCSW available at the clinic to train as the DE, so the effects of the clinician cannot be separated from the education intervention itself. As with all research studies, and primary care in general, a few participants were lost between the enrollment and the education visit $(n=27)$ and at onemonth follow-up $(n=18)$, however these losses were attributed to typical life circumstances (phone disconnected, patient relocated) and the overall study retention rate was quite high (87\%). Additionally, the outcome 
measure of engagement in depression treatment at one month follow up was a self-report item collected via phone, which introduces inaccuracy and potential response bias.

\section{Conclusions}

Interventions in primary care that aim to educate patients in culturally meaningful ways may facilitate early identification of barriers and result in better engagement in treatment, especially for disparity populations. Culturally appropriate tools which address person-level barriers can lead to active engagement of the patient and family members in shared decision-making regarding treatment and offer solutions for the patient to adhere to treatment, share information about response and side effects, and facilitate decision support [38, 43, 44]. Findings from the current study provided sufficient pilot data to lead to a National Institutes of Health funded rigorous evaluation of the fotonovela intervention in a randomized control trial, an important next step [45]. More research is needed on implementation of evidence-based guidelines at the provider and systems levels to improve treatment options and patient outcomes in the Hispanic community.

\begin{abstract}
Abbreviations
BA: Behavioral activation; CMS: Center for Medicare and Medicaid Services; DESEO: Depression Screening and Education: Options to Reduce Barriers to Treatment; DKM: Depression knowledge measure; FQHC: Federally qualified health center; GED: General Education Diploma; LCSW: Licensed Clinical Social Worker; LSAS: Latino Scale for Antidepressant Stigma; PHQ-9: 9-Item Patient Health Questionnaire; PI: Principal Investigator; SCMHC: Stigma Concerns about Mental Health Care; SD: Social Distance scale
\end{abstract}

\section{Acknowledgements}

The authors would like to thank the producers of the fotonovela, "Secret Feelings," Mel Baron, Pharm.D. and Gregory Molina from the University of Southern California School of Pharmacy.

The authors would also like to acknowledge the research assistants Monica Lechuga and Marisol Ada Vargas Vilugron for their assistance in collection of the data for Project DESEO. Additionally, the author would like to thank the social worker Depression Educator, Damaris Lopez, LCSW.

\section{Authors' contributions}

KS was the PI for Project DESEO, conceived and designed the study, and drafted the manuscript. MOK assisted in the design of the study and analyzed the data and interpreted the results. BHE was the Project Coordinator for Project DESEO and assisted in the drafting of the manuscript. $\mathrm{LC}$ was part of the development team for the Depression Education Fotonovela. MHT created the depression screening tool for use in primary care. All authors read and approved the final manuscript.

\section{Funding}

This study was funded by a grant from the U.S. Department of Health and Human Services Center for Medicare and Medicaid Services (CMS), Center for Medicare and Medicaid Innovation, Grants to Support the Hispanic Health Services Research Grant Program (Grant No. 1H0CMS331363-01-00). CMS had no role in the design of the study protocol, however this funding opportunity specified support for Educational Intervention Studies, which will inform populations-at-risk about certain health problems especially as they relate to Hispanics. CMS also required that the study reach a minimum of 350 participants.

\section{Availability of data and materials}

The datasets used and/or analyzed during the current study are available from the corresponding author on reasonable request.

\section{Ethics approval and consent to participate}

The DESEO study protocol was reviewed and approved by the Institutional Review Board of the University of Texas at Arlington (IRB reference number: 2015-0336). All patients provided written informed consent prior to participation in the study.

\section{Consent for publication}

Not applicable.

\section{Competing interests}

The authors declare that they have no competing interests.

\section{Author details}

${ }^{1}$ School of Social Work, University of Texas at Arlington, 211 South Cooper Street, Arlington, TX 76019, USA. ²Department of Psychiatry, UT Southwestern Medical Center, Dallas, TX, USA. ${ }^{3}$ College of Social Work, Florida State University, Tallahassee, FL, USA. ${ }^{4}$ George Warren Brown School of Social Work, Washington University in St. Louis, St. Louis, MO, USA.

Received: 31 December 2018 Accepted: 25 September 2019

Published online: 21 October 2019

\section{References}

1. WHO. Depression and Other Common Mental Disorders: Global Health Estimates. Geneva: World Health Organization; 2017.

2. Kessler RC, Aguilar-Gaxiola S, Alonso J, et al. The global burden of mental disorders: an update from the WHO world mental health (WMH) surveys. Epidemiol Psichiatr Soc. 2009;18(1):23-33.

3. Kessler RC. The costs of depression. The Psychiatric Clinics of North America. 2012;35(1):1-14

4. Scherrer JF, Garfield LD, Chrusciel T, et al. Increased risk of myocardial infarction in depressed patients with type 2 diabetes. Diabetes Care. 2011; 34(8):1729-34.

5. Sanchez K, Chapa T, Ybarra R, Martinez ON. Eliminating health disparities through culturally and linguistically centered integrated health care: consensus statements, recommendations, and key strategies from the field. J Health Care Poor Underserved. 2014;25(2):469-77.

6. Kaliebe K. The Future of Psychiatric Collaboration in Federally Qualified Health Centers. Psychiatr Serv. 2016;67(8).

7. Alegria M, Canino G, Shrout PE, et al. Prevalence of mental illness in immigrant and non-immigrant U.S. Latino groups. Am J Psychiatry. 2008; 165(3):359-69.

8. Alegria M, Mulvaney-Day N, Torres M, Polo A, Cao Z, Canino G. Prevalence of psychiatric disorders across Latino subgroups in the United States. Am J Public Health. 2007:97(1):68-75.

9. Li CY, Mokdad AH, Ford ES, Strine TW. Prevalence of depression among US adults with diabetes - findings from the 2006 behavioral risk factor surveillance system. Diabetes Care. 2008:31(1):105-7.

10. Interian A, Ang A, Gara MA, Rodriguez MA, Vega WA. The long-term trajectory of depression among Latinos in primary care and its relationship to depression care disparities. Gen Hosp Psych. 2011;33(2):94-101.

11. Gonzalez HM, Tarraf W, Whitfield KE, Vega WA. The epidemiology of major depression and ethnicity in the United States. J Psychiatr Res. 2010;44(15): 1043-51.

12. Cooper LA, Gonzales JJ, Gallo JJ, et al. The acceptability of treatment for depression among African-American, Hispanic, and white primary care patients. Med Care. 2003:41(4):479-89,

13. Vega WA, Kolody B, Aguilar-Gaxiola S, Catalano R. Gaps in service utilization by Mexican Americans with mental health problems. Am J Psychiatr. 1999; 156(6):928-34.

14. Cabassa L, Hansen MC, Palinkas LA, Ell K. Azucar y nervios: explanatory models and treatment experiences of Hispanics with diabetes and depression. Soc Sci Med. 2008;66(12):2413-24.

15. Ell K, Xie B, Kapetanovic S, et al. One-year follow-up of collaborative depression Care for low-Income, predominantly Hispanic patients with Cancer. Psychiatr Serv. 2011;62(2):162-70. 
16. Sanchez K, Ybarra R, Chapa T, Martinez ON. Eliminating behavioral health disparities and improving outcomes for racial and ethnic minority populations. Psychiatr Serv. 2016;67(1):13-5.

17. Martinez Pincay IE, Guarnaccia PJ. "It's like going through an earthquake": anthropological perspectives on depression among Latino immigrants. Journal of immigrant and minority health / Center for Minority Public Health. 2007;9(1):17-28.

18. Eghaneyan BH, Sanchez K, Killian M. Integrated health care for decreasing depressive symptoms in Latina women: initial findings. Journal of Latina/o Psychology. 2017;5(2):118-25.

19. Sanchez K, Eghaneyan BH, Trivedi MH. Depression screening and education: options to reduce barriers to treatment (DESEO): protocol for an educational intervention study. BMC Health Serv Res. 2016;16(1):1-9.

20. Kroenke K, Spitzer RL. The PHQ-9: a new depression diagnostic and severity measure. Psychiatr Ann. 2002;32(9):509-15.

21. Lopez V, Sanchez K, Killian MO, Eghaneyan BH. Depression screening and education: an examination of mental health literacy and stigma in a sample of Hispanic women. BMC Public Health. 2018;18(1):018-5516.

22. Cabassa LJ, Molina GB, Baron M. Depression fotonovela: development of a depression literacy tool for Latinos with limited English proficiency. Health Promot Pract. 2012;13(6):747-54.

23. Cabassa LJ. In: Schwartz SJ, Unger J, editors. Depression Fotonovela: integrating cultural factors and entertainment-education to improve depression literacy in the Latino community. Oxford University Press: The Oxford Handbook of Acculturation and Health; 2016.

24. Hernandez MY, Organista KC. Entertainment-education? A Fotonovela? A New Strategy to Improve Depression Literacy and Help-Seeking Behaviors in At-Risk Immigrant Latinas. American Journal of Community Psychology. 2013;52:224-35

25. Unger JB, Cabassa LJ, Molina GB, Contreras S, Baron M. Evaluation of a Fotonovela to increase depression knowledge and reduce stigma among Hispanic adults. J Immigr Minor Health. 2013;15(2):398-406.

26. American Psychiatric Association. Diagnostic and Statistical Manual of Mental Disorders DSM-IV. 4th ed. Washington. D.C: American Psychiatric Association; 1994.

27. Huang FY, Chung H, Kroenke K, Delucchi KL, Spitzer RL. Using the patient health questionnaire-9 to measure depression among racially and ethnically diverse primary care patients. J Gen Intern Med. 2006:21(6):547-52.

28. Interian A, Ang A, Gara MA, Link BG, Rodriguez MA, Vega WA. Stigma and depression treatment utilization among Latinos: utility of four stigma measures. Psychiatr Serv. 2010;61(4):373-9.

29. Link BG, Phelan JC, Bresnahan M, Stueve A, Pescosolido BA. Public conceptions of mental illness: labels, causes, dangerousness, and social distance. Am J Public Health. 1999;89(9):1328-33.

30. Martin JK, Pescosolido BA, Tuch SA. Of Fear and Loathing: The Role of 'Disturbing Behavior,' Labels, and Causal Attributions in Shaping Public Attitudes toward People with Mental IInness. J Health Soc Behav. 2000;41(2):208-23.

31. U.S. Department of Commerce. Texas QuickFacts from the US Census Bureau. United States Census Bureau. Quickfacts.census.gov. Published 2011. Accessed.

32. Wu SY, Ell K, Gross-Schulman SG, et al. Technology-facilitated depression care management among predominantly Latino diabetes patients within a public safety net care system: comparative effectiveness trial design. Contemp Clin Trials. 2014;37(2):342-54.

33. Bauer AM, Schillinger D, Parker MM, et al. Health literacy and antidepressant medication adherence among adults with diabetes: the diabetes study of northern California (DISTANCE). J Gen Intern Med. 2013;28(9):1181-7.

34. Kelly K, Posternak M, Alpert JE. Toward achieving optimal response: understanding and managing antidepressant side effects. Dialogues Clin Neurosci. 2008;10(4):409-18.

35. Valle R, Yamada AM, Matiella AC. Fotonovelas: a health literacy tool for educating Latino older adults about dementia. Clin Gerontol. 2006;30(1):71-88.

36. Wilkin HA, Valente TW, Murphy S, Cody MJ, Huang G, Beck V. Does entertainment-education work with Latinos in the United States? Identification and the effects of a telenovela breast cancer storyline. J Health Commun. 2007;12(5):455-69.

37. Diaz V. Encouraging participation of minorities in research studies. Ann Fam Med. 2012 Jul-Aug;10(4):372-373. doi: https://doi.org/10.1370/afm.1426.; 2012.

38. Dixon L, Lieberman J. Psychiatry Embraces Patient-Centered Care. Psychiatric News. 2014. Published 7 Feb 2014.
39. Dwight-Johnson M, Sherbourne CD, Liao D, Wells KB. Treatment preferences among depressed primary care patients. J Gen Intern Med. 2000;15(8):527-34.

40. Clever SL, Ford DE, Rubenstein LV, et al. Primary care patients' involvement in decision-making is associated with improvement in depression. Med Care. 2006;44(5):398-405.

41. Coleman K, Austin BT, Brach C, Wagner EH. Evidence on the Chronic Care Model in the new millennium. Health Affairs Web site. $<$ Go to $|S|>: / /$ 000262194400011. Published 2009. Updated Jan-Feb. Accessed 1, 28.

42. Stacey D, Legare F, Col NF, et al. Decision aids for people facing health treatment or screening decisions. Cochrane Database Syst Rev. 2014;1:335.

43. Barry MJ, Edgman-Levitan S. Shared decision making - the pinnacle of patient-centered care. N Engl J Med. 2012;366(9):780-1.

44. Cohen DJ, Balasubramanian BA, Isaacson NF, Clark EC, Etz RS, Crabtree BF. Coordination of health behavior counseling in primary care. Ann Fam Med. 2011;9(5):406-15.

45. Sanchez K, Eghaneyan BH, Killian MO, Cabassa L, Trivedi MH. Measurement, education and tracking in integrated care (METRIC): use of a culturally adapted education tool versus standard education to increase engagement in depression treatment among Hispanic patients: study protocol for a randomized control trial. Trials. 2017;18(1):017-2109.

\section{Publisher's Note}

Springer Nature remains neutral with regard to jurisdictional claims in published maps and institutional affiliations.
Ready to submit your research? Choose BMC and benefit from:

- fast, convenient online submission

- thorough peer review by experienced researchers in your field

- rapid publication on acceptance

- support for research data, including large and complex data types

- gold Open Access which fosters wider collaboration and increased citations

- maximum visibility for your research: over $100 \mathrm{M}$ website views per year

At BMC, research is always in progress.

Learn more biomedcentral.com/submissions 